\title{
Pelvic Rhabdomyosarcoma
}

National Cancer Institute

\section{Source}

National Cancer Institute. Pelvic Rhabdomyosarcoma. NCI Thesaurus. Code C151981.

Rhabdomyosarcoma involving the organs and structures in the pelvis. 